\title{
Heparin for Vertebral Intraluminal Thrombus Causing Retroperitoneal Hemorrhage from Occult Renal Angiomyolipoma
}

\author{
Billie Hsieh ${ }^{a} \quad$ Muhammad B. Tariq $^{a}$ Lamya Ibrahim $^{a}$ \\ Shekhar D. Khanparab Larry A. Kramer ${ }^{b}$ Sean I. Savitz ${ }^{a}$ \\ anstitute for Stroke and Cerebrovascular Disease, The University of Texas Health Science \\ Center, Houston, TX, USA; bepartment of Radiology, The University of Texas Health \\ Science Center, Houston, TX, USA
}

\section{Keywords}

Stroke - Ischemic · Heparin · Atrial fibrillation arrhythmia - Hemorrhage · Tumor subject

terms · Ischemic stroke $\cdot$ Imaging $\cdot$ Blood pressure $\cdot$ Atrial fibrillation $\cdot$ Arrhythmias

\begin{abstract}
Stroke is a common cause of mortality and serious long-term disability worldwide. In the acute setting, current American Heart Association/American Stroke Association guidelines do not recommend routine anticoagulation for the management of acute ischemic strokes. However, short-term use of unfractionated heparin (UFH) in select subpopulations has demonstrated improved outcomes. While tools such as CHADSVASC and HASBLED scores are useful in stratifying risk of long-term anticoagulation in patients with nonvalvular atrial fibrillation and additional risk factors, the carefully selected patient populations for the design of these studies do not account for risk of hemorrhage from other preexisting conditions. Here, we present a patient with a posterior circulation intraluminal thrombus treated with UFH, who manifested with a near-fatal intra-abdominal hemorrhage from a previously undetected renal angiomyolipoma (AML).
\end{abstract}

\section{Introduction/Background}

Approximately 795,000 people suffer from a stroke every year in the USA; $87 \%$ of which are ischemic, while the remaining majority are due to intracranial or subarachnoid hemorrhages [1]. Proposed benefits for the use of heparin in acute ischemic strokes include early 
clot lysis, stopping propagation of the clot, reduction in blood viscosity and recurrent thromboembolism, as well as possible anti-inflammatory properties [2-4]. The timing of anticoagulation initiation in the early phase is debatable [5]. Heparin use can be complicated by hemorrhage in $2-3 \%$ of patients or even higher percentages depending on the clinical case [6]. Bleeding can potentially occur from any site, but gastrointestinal bleeds are most common, and intracranial hemorrhages are the most dreaded. A case-by-case review of individual patients prior to starting anticoagulation can mitigate this risk [7, 8].

\section{Case Presentation}

The patient is a 70-year-old man with a medical history of hypertension, gout, and rectal cancer with liver metastases who presented with 4 days of nausea, vomiting, and gait instability. On physical examination, he was found to have right ptosis, left arm tremor, and right arm dysmetria. CT head showed cerebellar infarction with surrounding cerebral edema in the territory of the right posterior inferior cerebellar artery (PICA). CT angiography of the head and neck showed right vertebral thrombus with distal reconstitution from retrograde flow along with moderate-to-severe stenosis in his bilateral posterior cerebral arteries. He was not a candidate for thrombolysis with tissue plasminogen activator or endovascular intervention being outside of the thrombectomy treatment window.

Stroke workup was completed, with a normal transthoracic echocardiogram, elevated hemoglobin A1c, and elevated LDL. He received an aspirin $325 \mathrm{mg}$ load and then was started on aspirin $81 \mathrm{mg}$ and a statin for secondary stroke prophylaxis. His blood pressure was slowly decreased from permissive hypertension with his home antihypertensives. MRI brain confirmed a subacute stroke of the right PICA territory with secondary right tonsillar herniation and no hemorrhagic transformation (Fig. 1). Mass effect was also seen with compression of the fourth ventricle without hydrocephalus. He further underwent MR angiography of his head and neck, which was significant for occlusion of his right vertebral artery from V1 to the distal V2 segment, with distal reconstitution. That is, trickle flow at the V3 segment level and normal caliber at $\mathrm{V} 4$ reflecting clot burden and likely retrograde flow from the basilar artery.

Intravenous heparin infusion without bolus was started for the right vertebral thrombus with therapeutic partial thromboplastin time reached on the second day of admission. The patient began complaining of abdominal pain on the third day, with recurrence of nausea and vomiting. He became hypotensive and had tachycardia. Hemoglobin dropped from 9.4 to 5.9 $\mathrm{mmol} / \mathrm{L}$. Abdominal X-ray showed possible ileus, with bowel diameter $>10 \mathrm{~cm}$. CT abdomen showed left retroperitoneal hematoma from a ruptured $1.2 \mathrm{~cm}$ renal angiomyolipoma (mildly complex and posterior). Aspirin and heparin were stopped. He was given 3 units of packed red blood cells (PRBCs) and emergently taken for mesenteric/visceral angiography with embolization by interventional radiology (IR) (Fig. 2).

Post-procedurally, he was initially hemodynamically stable but had new-onset atrial fibrillation with rapid ventricular response to the 160 s on telemetry and his systolic blood pressure dropped to $70 \mathrm{~mm} \mathrm{Hg}$. Repeat angiography of the embolized site was reviewed with IR. There was no active extravasation. He was given $1 \mathrm{~L}$ of fluids, 2 units of PRBCs along with amiodarone bolus and then started on an amiodarone infusion which was stopped the next day. Repeat electrocardiograms showed sinus rhythms. His hemoglobin was repeated every 8-12 h, then daily. Due to a high CHADSVASC score of 5, it was felt strongly that he needed to be on antithrombotic therapy. Repeat CT abdomens showed a stable hematoma; thus, he was restarted on aspirin $81 \mathrm{mg}$, 4 days after his embolization.

The case was further discussed with IR, oncology, hematology, surgery, gastrointestinal, and urology. The decision was that the benefits of anticoagulation outweighed the risks of

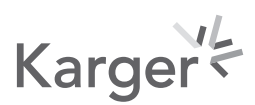




\section{Case Reports in \\ Neurology}
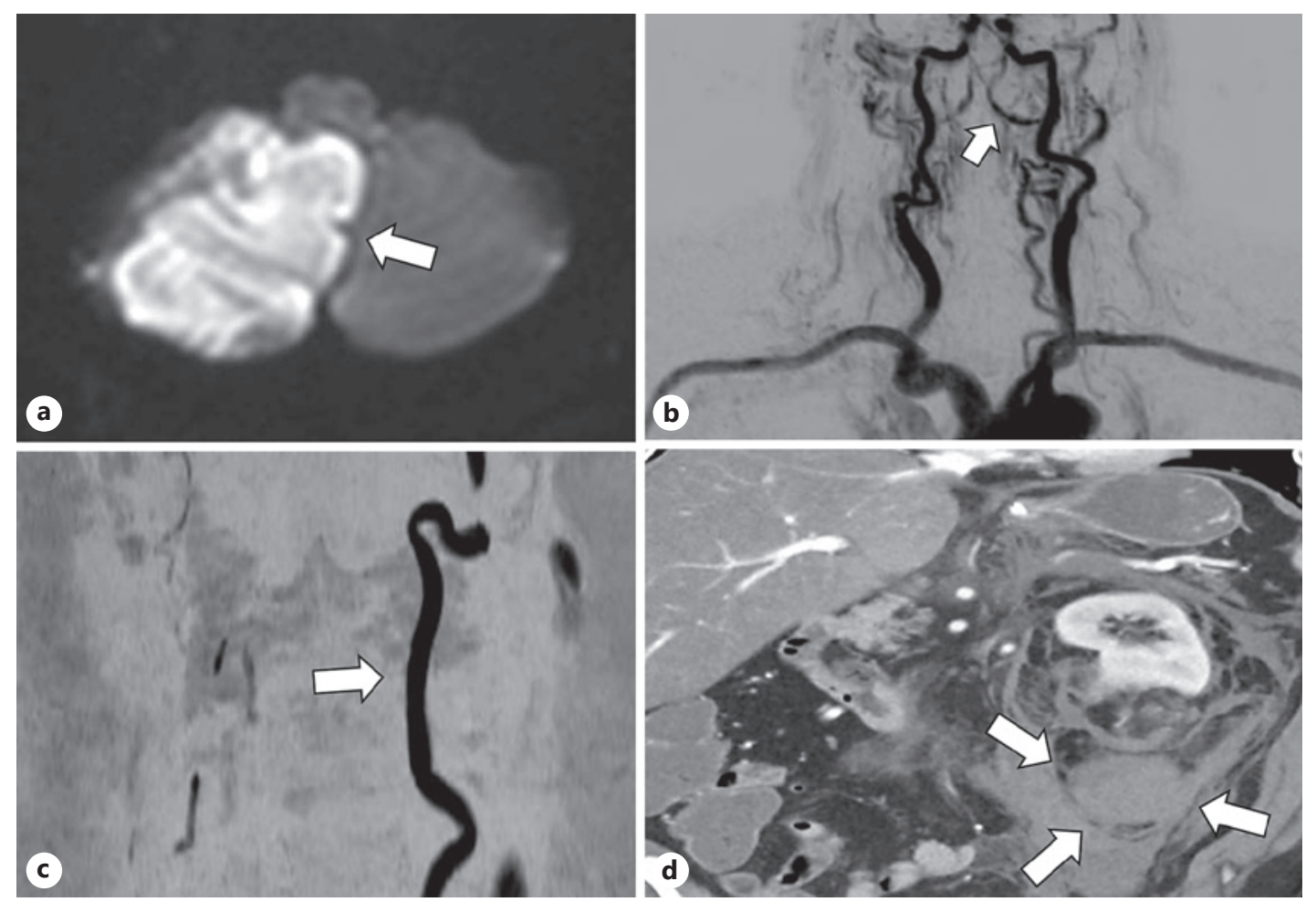

Fig. 1. a Restricted diffusion in the right posteromedial inferior cerebellum (arrow) compatible with a right PICA subacute infarction. No hematoma is identified. $\mathbf{b}$ Early phase of a reconstructed dynamic contrast MRA brain and neck. The left vertebral artery is well visualized throughout its course. There is some reflux of contrast in the right vertebral artery from the left vertebral artery (arrow). c Noncontrast MRA set to display flow in the rostral direction. Near the craniovertebral junction, only flow in the left vertebral artery is seen (arrow). d CT abdomen axial showing a defect in the lower pole of the left kidney containing fat density measuring $<4 \mathrm{~cm}$ in diameter (AML) with contiguous large retroperitoneal hemorrhage (arrows). PICA, posterior inferior cerebellar artery.

rebleeding after embolization. His hemoglobin remained stable. On day 7 after embolization, he was started on an aspirin to warfarin bridge. Device implantation or atrial ablation for atrial fibrillation management was not selected due to patient preference. At this point, he was discharged from the hospital with home health and close multispecialty follow-up. At 6 months follow-up, he was on anticoagulation and functioning close to his baseline at home.

\section{Discussion}

We present a case of a stroke patient who needed anticoagulation for a vertebral artery thrombus which had resulted in cerebellar infarction. Given the vertebral thrombus, the patient was at risk of further propagation into a brainstem stroke. Brainstem strokes are by themselves associated with a high mortality of 17\% [9] and cerebellar strokes with a mortality of $23 \%$. Given the potentially devastating complications, he was started on unfractionated heparin to aid in clot dissolution, which was partially achieved on repeat imaging. However, the patient bled due to an unknown AML. The dilemma in this case is the unexpected pathology.

Extracranial bleeding on heparin is uncommon (3\%), and only a small proportion of bleeding on anticoagulation is associated with an occult lesion [10-12]. Thus, routine 


\section{Case Reports in Neurology}

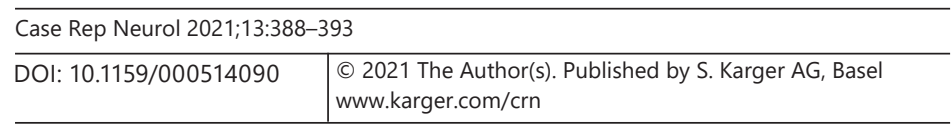

Hsieh et al:: AML Hemorrhage from Heparin for Vertebral Thrombus

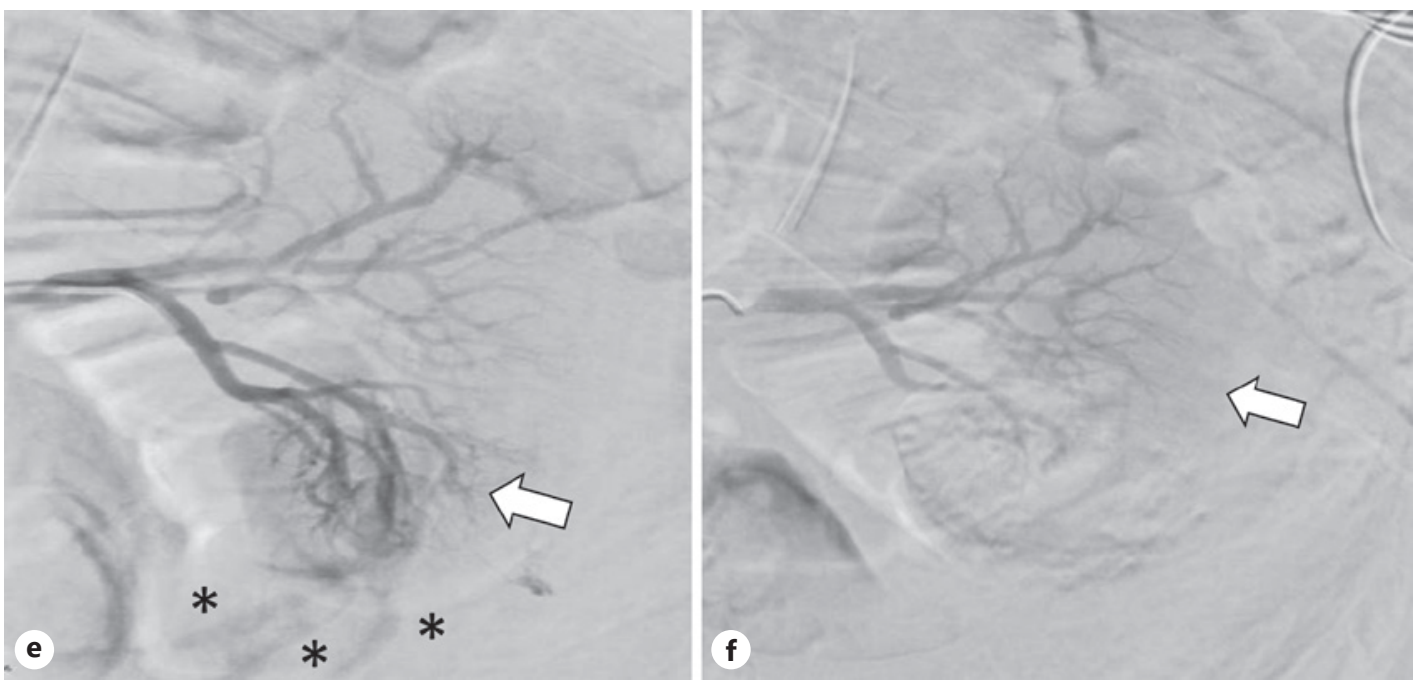

Fig. 2. e Pre-embolization arterial phase angiogram showing a hypervascular blush (arrow) in the right inferior renal pole with active contrast extravasation extending inferior to the kidney in multiple streams (asterisks). $\mathbf{f}$ Post-embolization arterial phase angiogram showing normal vascularization of the upper pole of the left kidney and hypovascularity of the lower pole (arrow) without active contrast extravasation.

abdominal imaging prior to starting anticoagulation is not commonly practiced. AML is a rare tumor, with a prevalence of $0.2-0.6 \%$ in the general adult population [13]. Hence, data on the number of bleeds from these occult tumors after starting anticoagulation is limited. Furthermore, there is no evidence on withholding anticoagulation even if an incidental AML is identified.

After the AML bled and was embolized, this patient went into atrial fibrillation, another condition associated with recurrent strokes. Paroxysmal atrial fibrillation could have gone previously undetected, with provoking factors being significant hypotension and related stressors, and the arrhythmia in turn causing a second episode of shock. Atrial fibrillation typically requires long-term anticoagulation [14]. With a high CHADSVASC score of 5, the risk of stroke, transient ischemic attack, or systemic embolism is as high as $10 \%$ per year [15]. Anticoagulation can decrease this risk by two-thirds and should be considered in all patients with high CHADSVASC scores [16]. However, the patient's HASBLED score was 3, which shows a 5.8\% risk for major bleeding [17]. Additionally, while there is no data for risk of AML bleeding on anticoagulation, or on whether anticoagulation would increase the inherent risk of bleeding, long-term follow-up of patients after embolization of their AMLs shows a rebleeding risk of $11-15 \%$ [18].

In this situation with high risk of future strokes and devastating nature of posterior circulation ischemic strokes sans treatment, while concurrently weighing a high risk of major hemorrhage with treatment, it is important to involve the patient in the decision-making process. The other critical aspect is early involvement of multiple subspecialties for their anecdotal input in such cases where no large studies exist.

\section{Statement of Ethics}

Written informed consent was obtained from the patient for publication of this case report and any accompanying images. 


\section{Conflict of Interest Statement}

The authors have no conflicts of interest to disclose.

\section{Funding Sources}

No sources of funding to disclose.

\section{Author Contributions}

Billie Hsieh MD wrote/edited the manuscript and created the figure, Muhammad B. Tariq MD co-wrote and edited the manuscript, Lamya Ibrahim MD co-wrote and edited the manuscript, Shekhar D. Khanpara MD helped create the figure, Larry A. Kramer MD helped create the figure and legend, and Sean I. Savitz MD edited the manuscript.

\section{References}

1 Virani Salim S, Alonso A, Benjamin Emelia J, Bittencourt Marcio S, Callaway Clifton W, Carson April P, et al. Heart disease and stroke statistics-2020 update: a report from the american heart association. Circulation. 2020;141(9):e139-e596.

2 Ruff IM, Jindal JA. Use of heparin in acute ischemic stroke: is there still a role? Curr Atheroscler Rep. 2015; 17(9):51.

3 Mokin M, Kass-Hout T, Kass-Hout O, Radovic V, Siddiqui AH, Levy EI, et al. Intravenous heparin for the treatment of intraluminal thrombus in patients with acute ischemic stroke: a case series. J Neurointerv Surg. 2013;5(2):144-50.

4 Rocha EA, Ji R, Ay H, Li Z, Arsava EM, Silva GS, et al. Reduced ischemic lesion growth with heparin in acute ischemic stroke. J Stroke Cerebrovasc Dis. 2019;28(6):1500-8.

5 Shahpouri MM, Mousavi S, Khorvash F, Mousavi SM, Hoseini T. Anticoagulant therapy for ischemic stroke: a review of literature. J Res Med Sci. 2012;17(4):396-401.

6 Onishi A, St Ange K, Dordick JS, Linhardt RJ. Heparin and anticoagulation. Front Biosci. 2016;21:1372-92.

7 Lip GY, Nieuwlaat R, Pisters R, Lane DA, Crijns HJ. Refining clinical risk stratification for predicting stroke and thromboembolism in atrial fibrillation using a novel risk factor-based approach: the euro heart survey on atrial fibrillation. Chest. 2010;137(2):263-72.

8 Pisters R, Lane DA, Nieuwlaat R, de Vos CB, Crijns HJ, Lip GY. A novel user-friendly score (HAS-BLED) to assess 1-year risk of major bleeding in patients with atrial fibrillation: the Euro heart survey. Chest. 2010;138(5): 1093-100.

9 Macdonell RA, Kalnins RM, Donnan GA. Cerebellar infarction: natural history, prognosis, and pathology. Stroke. 1987;18(5):849-55.

10 Zidane M, Schram MT, Planken EW, Molendijk WH, Rosendaal FR, van der Meer FJ, et al. Frequency of major hemorrhage in patients treated with unfractionated intravenous heparin for deep venous thrombosis or pulmonary embolism: a study in routine clinical practice. Arch Intern Med. 2000;160(15):2369-73.

11 The International Stroke Trial (IST): a randomised trial of aspirin, subcutaneous heparin, both, or neither among 19435 patients with acute ischaemic stroke. International Stroke Trial Collaborative Group. Lancet. 1997;349(9065):1569-81.

12 Flack KF, Desai J, Kolb JM, Chatterjee P, Wallentin LC, Ezekowitz M, et al. Major gastrointestinal bleeding often is caused by occult malignancy in patients receiving warfarin or dabigatran to prevent stroke and systemic embolism from atrial fibrillation. Clin Gastroenterol Hepatol. 2017;15(5):682-90.

13 Vos N, Oyen R. Renal angiomyolipoma: the good, the bad, and the ugly. J Belg Soc Radiol. 2018;102(1):41.

14 Wolf PA, Dawber TR, Thomas HE Jr, Kannel WB. Epidemiologic assessment of chronic atrial fibrillation and risk of stroke: the Framingham study. Neurology. 1978;28(10):973-7.

15 Risk factors for stroke and efficacy of antithrombotic therapy in atrial fibrillation. Analysis of pooled data from five randomized controlled trials. Arch Intern Med. 1994;154(13):1449-57.

16 Friberg L, Rosenqvist M, Lip GY. Evaluation of risk stratification schemes for ischaemic stroke and bleeding in 182,678 patients with atrial fibrillation: the Swedish atrial fibrillation cohort study. Eur Heart J. 2012;33(12): 1500-10. 
17 Lip GY, Frison L, Halperin JL, Lane DA. Comparative validation of a novel risk score for predicting bleeding risk in anticoagulated patients with atrial fibrillation: the HAS-BLED (hypertension, abnormal renal/liver function, stroke, bleeding history or predisposition, labile inr, elderly, drugs/alcohol concomitantly) score. J Am Coll Cardiol. 2011;57(2):173-80.

18 Andersen PE, Thorlund MG, Wennevik GE, Pedersen RL, Lund L. Interventional treatment of renal angiomyolipoma: immediate results and clinical and radiological follow-up of 4.5 years. Acta Radiol Open. 2015;4(7): 2058460115592442. 